\title{
64 x 64 Pixels General Purpose Digital Vision Chip
}

\author{
Takashi Komuro and Masatoshi Ishikawa
}

Department of Information Physics and Computing,Graduate School of Information Science and Engineering, The University of Tokyo, Japan (e-mail: kom@k2.t.u-tokyo.ac.jp)

\begin{abstract}
Conventional image processing has a critical limit of the frame rate, which is a result of serial transmission of the video signal. In order to overcome this limit, a vision chip in which photo detectors and parallel processing elements are integrated together has been proposed. In this paper, the general purpose vision chip with digital processing elements and the $64 \times 64$ pixels prototype chip we developed will be described.
\end{abstract}

Key words: vision chip, image sensor, parallel processing, visual feedback

\section{INTRODUCTION}

The expectation for real-time sensory information processing is increasing in the field of robot vision, intelligent transportation systems, high speed inspection, and so on. High speed visual feedback control is especially useful in performing advanced tasks in the real world. A feedback rate of over $1 \mathrm{kHz}$ makes maximum use of actuator performance.

However, conventional vision systems use serial transmission of video signals from an image sensor such as CCD to a processing device such as DSP. In such a system, the frame rate is limited by the video signal such as NTSC (33ms).

On the other hand, a device called a vision chip has been proposed, in which photo detectors (PDs) and processing elements (PEs) are connected in each pixel. This chip captures and processes images in pixel-parallel without serial transmission. Therefore, this chip achieves high speed visual feedback at over $1 \mathrm{kHz}$. Also, by integrating a sensor and a processor together in one chip, we expect benefits of being a system VLSI such as compactness, low cost, and low power.

The original version of this chapter was revised: The copyright line was incorrect. This has been corrected. The Erratum to this chapter is available at DOI: 10.1007/978-0-387-35597-9_40 


\section{DIGITAL VISION CHIP}

There has been considerable research on vision chips, but most of them use analog circuits in the processing element[1]. Analog circuits require less area and therefore are easier to integrate than digital ones. However, they have a drawback in that the functions are few and almost fixed once they are designed. To make a general purpose vision chip that can process various algorithms in a changing environment, it is necessary to develop a programmable processing element that can be controlled by software. Digital circuits are effective in accomplishing this purpose. We call such vision chips containing digital circuits "digital vision chips".

Considering the above mentioned requirements, Ishikawa et al. proposed an architecture for a digital vision chip[2]. Using a scaled-up model of the vision chip, various application systems have been developed[3-6]. These systems have achieved high speed visual feedback at a sampling period of $1 \mathrm{~ms}$ and have achieved very high speed real-time control that can't be achieved in existing systems.

Research on digital vision chips has been carried out at a few other laboratories. In France, a compact digital vision chip called the programmable artificial retina has been proposed[7], and $65 \times 76$ pixels and $128 \times 128$ pixels chips have been developed. In Sweden a unique digital vision chip based on their near-sensor image processing concept was proposed and a 32x32 pixel chip has been developed[8].

One of the problems in designing a digital vision chip is that it is difficult to integrate many pixels on a single chip since the digital circuits requires more area than analog circuits.

To integrate a large number of pixels in one chip, we have to make an effort to design a compact PE whose area is as small as possible.

\section{BASIC ARCHITECTURE}

Following the design concept descrived above, Komuro et al. designed a new architecture $S^{3} \mathrm{PE}$ (Simple and Smart Sensory Processing Element) [9] with the goal of further high integration. Figure 1 shows the architecture of $S^{3} \mathrm{PE}$.

Each PE has an ALU, which has bit-serial operation structure and 24 bit local memory in which each bit can be randomly accessed. It communicates with the photo detector and the neighbor PEs via an 8 channel I/O which is mapped to memory address space. 


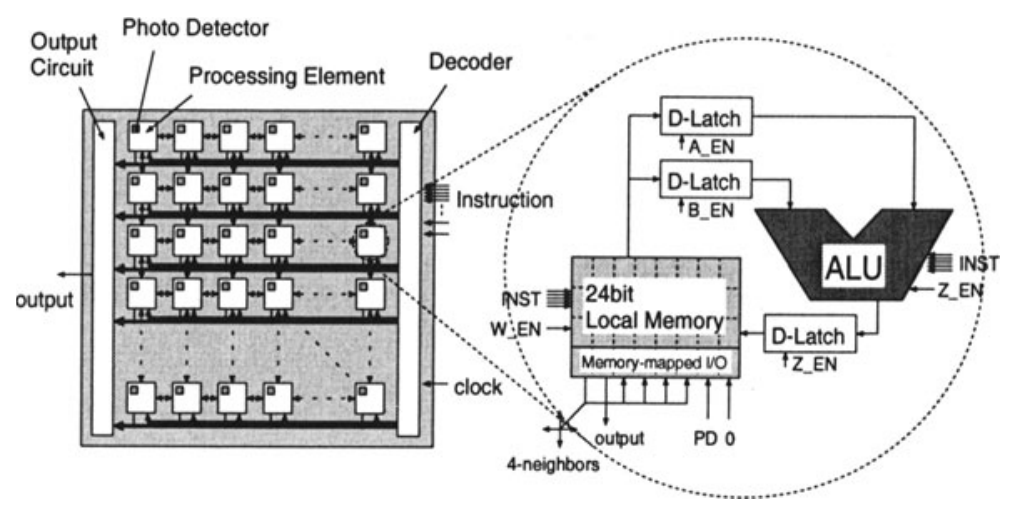

Figure 1. Basic Architecture of General Purpose Vision Chip

The ALU has a simple structure consisting of a full-adder, a carry register and some multiplexers. It can process one of 10 different kinds of logical and 8 kinds of arithmetic operations at each cycle. Bit-serial operation is used that the ALU performs each bit of multi-bit operations in order. This is slower than bit-parallel operation, but has the advantage of compact circuitry and the capability of variable bit length data operation

In the local memory, 24 bit random access memory (RAM) and 8 bit I/O ports are allocated to the same address space. I/O ports are connected to up, down, left and right PEs, input from the sensor, and zero signal. With the introduction of the memory mapped $\mathrm{V} / \mathrm{O}$, all processing, including not only load/store operations but also $\mathrm{V} / \mathrm{O}$ operations are performed by accessing the local memory.

As a result, the instruction code has a simple form : all instructions consist of read address A, B (5 bits each), operation code (5 bits), and write address ( 5 bits). This 20 bit instruction is divided into four steps, and is transmitted and processed in order. This vision chip can process most of the early vision algorithms such as edge detection, smoothing, and convolution filtering in the order of a $\mu \mathrm{s}$, which is much faster than conventional image processing systems and is fast enough for visual feedback control of a robot. Gray-scale image processing algorithms can also be implemented as well as binary ones since the PE has enough memory and arithmetic operation function. This is a big advantage to the other existing chips mentioned above. Tablel shows the steps and processing time. The cycle time per instruction is supposed to be $100 \mathrm{~ns}$. Practically, to achieve this speed, a controller which can transmit control signals to the vision chip at very high speed is needed. 
Table 1. Steps and Processing Time of Early Vision Algorithms

\begin{tabular}{|l|c|l|}
\hline Algorirhm & steps & time \\
\hline Edge Detection (binary) & 8 & $0.8 \mu \mathrm{s}$ \\
Smoothing (binary) & 14 & $1.4 \mu \mathrm{s}$ \\
Edge Detection (6bit) & 47 & $4.7 \mu \mathrm{s}$ \\
Smoothing (6bit) & 41 & $4.1 \mu \mathrm{s}$ \\
Thinning (binary) $*$ & 12 & $1.2 \mu \mathrm{s}$ \\
Convolution (6bit) & 986 & $99 \mu \mathrm{s}$ \\
Poisson Equation (6bit) * & 65 & $6.5 \mu \mathrm{s}$ \\
\hline
\end{tabular}

* repeating operation

\section{BASIC ARCHITECTURE}

In addition to the effort in designing the compact architecture, the rapid progress of semiconductor integration technology in recent years has enabled development of a general purpose digital vision chip with a large number of pixels. Our first goal is to develop a general purpose vision chip with $64 \times 64$ pixels, which is considered to be the minimum requirement for many industrial applications.

Based on the designed architecture, we have developed some test chips with $8 \times 8$ pixels[9] and 16x16 pixels[10]. Also we developed a $64 \times 64$ pixels chip but it has some defects in memory access and photo detector sensitivity. Then, we re-designed the circuit. We made some modifications as described below and realized further integration.

\section{Global wiring of the control line}

In a multi-layer process, the area taken up by wires is smaller than that of transistors. In this design, the decoders of control signal and address signals are taken out of the PE. All signals are transmitted to the PE after they are decoded. Those global wires are placed not in the exclusive area but over the circuits.

\section{Single bit line SRAM}

Usually, an SRAM has two bit lines - bit signal and its compliment. Writing data is performed by giving an input signal and its complement respectively to the bit lines, and reading data is performed by taking a differential of two bit lines using a sense amp. This structure is adopted mainly in order to solve the problem of speed. Many SRAM elements are connected to a bit line and the parasitic capacitance of the line is so large that an SRAM element can't rapidly reverse the value. 
However, in the vision chip, area has priority over speed. Also only four SRAMs are connected to a bit line. Moreover, the power consumption in the sense amp has been an obstacle against further integration. Therefore, we adopted single line SRAM.

Consequently, the SRAM elements are small, the row-select multiplexers of the memory are half size, and the sense amp is removed.

In former designs, the $\mathrm{AD}$ conversion in the $\mathrm{PD}$ is performed by counting time until output of an inverter is reversed. In this design, the inverter is replaced by a comparator and the voltage is compared to the reference voltage $\mathrm{V}_{\text {th }}$.

The circuit using the inverter is smaller than that using the comparator, but there is a problem in that direct-current flows at the time around the inverter's reversal and the total power consumption is enormous. The circuit using the comparator has an advantage in that power consumption is reduced considerably and the threshold voltage is changeable in $\mathrm{AD}$ conversion. Figure 2 shows the photo detector circuit and the method of $\mathrm{AD}$ conversion. The slope of the voltage of the comparator input is steep in proportion to the light intensity and the time until it reaches the threshold is short.

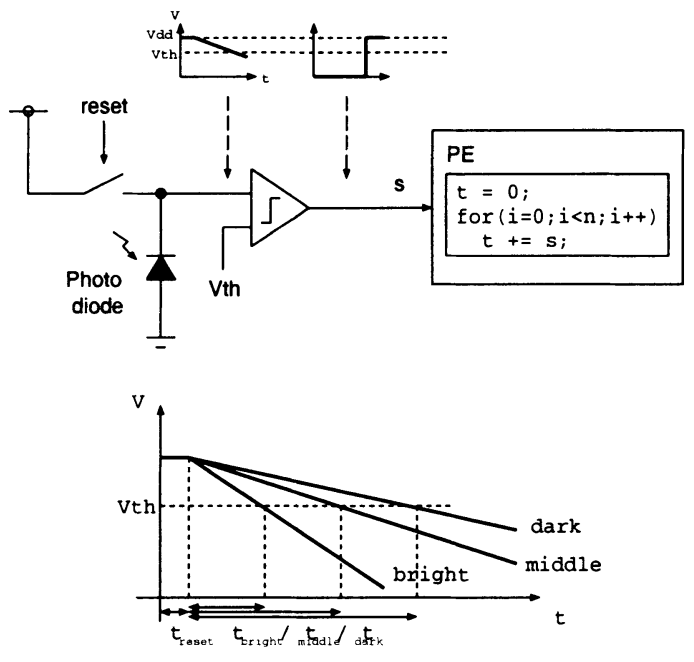

Figure 2. Photo Detector and AD Conversion 
In addition to these modifications, some new functions including global feature extraction are introduced in this design but they are not described in this paper.

The layout of the chip has $64 \times 64$ pixels in a $5.4 \mathrm{~mm} \times 5.4 \mathrm{~mm}$ area using $0.35 \mu \mathrm{m}$ TLM CMOS process. The number of transistors per PE is about 400 and the PE area is as small as $67.4 \mu \mathrm{m} \times 67.4 \mu \mathrm{m}$. This means that $256 \times 256$ pixels can be integrated in about a $1.8 \mathrm{~cm} \times 1.8 \mathrm{~cm}$ chip and the standard pixel number as an image processing device is achievable. Figure 3 shows the layout of the pixel. It is confirmed that the chip works at least with the control speed of $10 \mathrm{MHz}$.

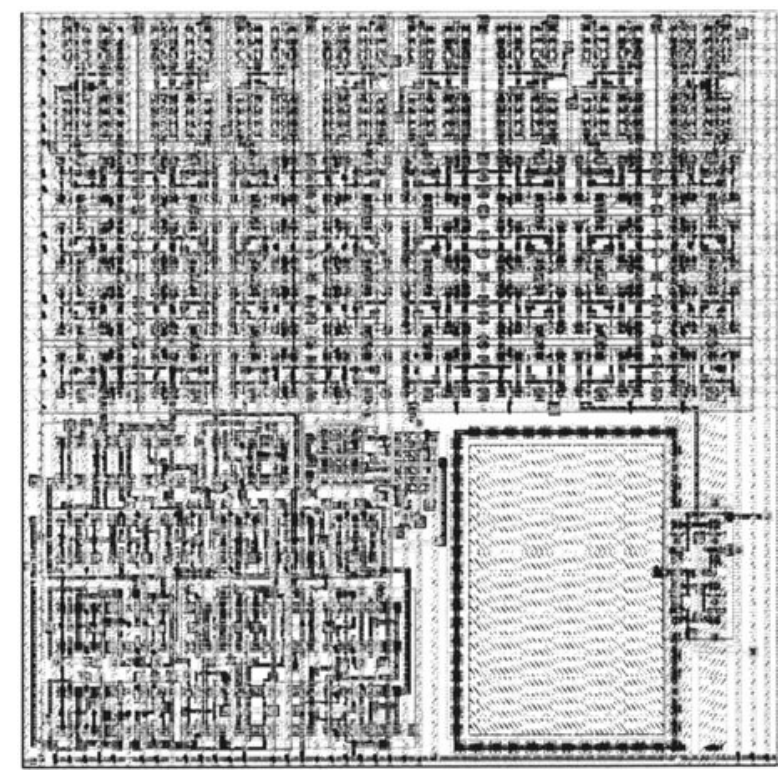

Figure 3. Layout of the Pixel

At that control speed, the cycle time per instruction is $2.2 \mu$ s. It seems to be slow but the potential peformance of the vision chip is much higher. At present the working speed is limited by the performance of the controller which transmit control signals to the vision chip. With faster controller the working speed will be greatly improved. Now we are developing such a controller. 


\section{BASIC ARCHITECTURE}

Using this design, we have developed a prototype chip of $64 \times 64$ pixels. Figure 4 shows a photograph of the chip. This chip is fabricated via CMP in France.

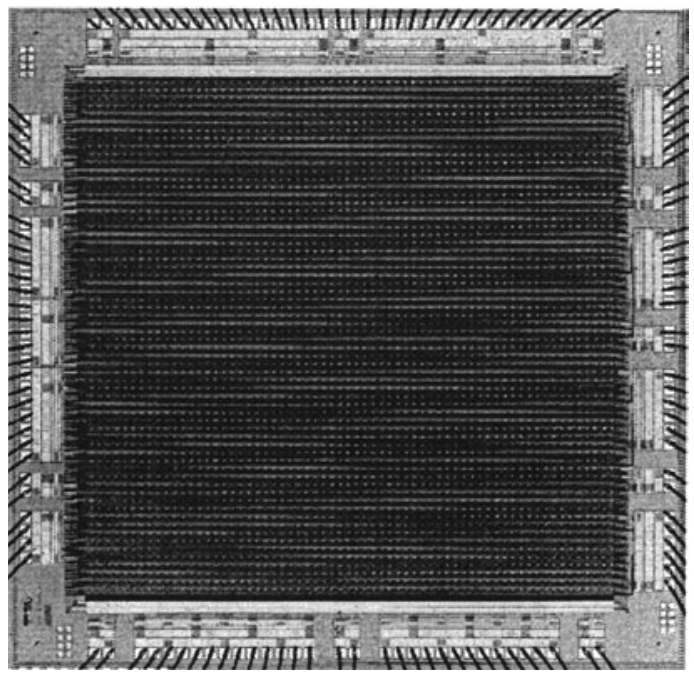

Figure 4. Photograph of General Purpose Vision Chip

Using this chip, experiments in image capturing and some processing with a lens attached were performed. The result is shown in Figure 5. In (a) the chip is irradiated directly by a spherical light source. In (b) a human hand was irradiated. The images were captured in 4bit gray-scale. In (c) an image of the same light was binarized and edge-detected. The integration time is $2.8 \mathrm{~ms}$. The threshold voltage of the $\mathrm{AD}$ conversion $\mathrm{V}_{\mathrm{t}}$ is $1.5 \mathrm{~V}$ in (a) and (c), and $3.3 \mathrm{~V}$ in (b). The higher the $\mathrm{V}_{\mathrm{t}}$ is, the higher the sensitivity of the PD becomes but the lower the quality of the image $(\mathrm{S} / \mathrm{N})$ becomes. 


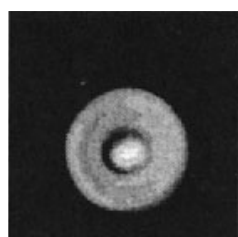

(a)

(b)
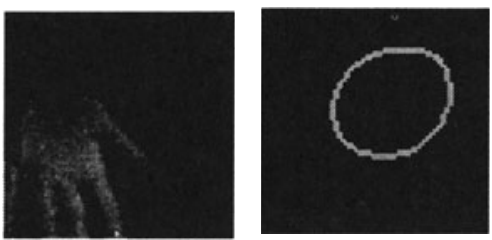

(c)

Figure 5. Sample Output

We did an experiment to evaluate the performance of the sensor. The chip surface was flatly irradiated by an LED array lighting without a lens. Figure 6 shows the graph of maximum, average, and minimum value in a screen when the image was captured in 8 bit gray-scale and $\mathrm{V}_{\mathrm{t}}$ was set to $2.4 \mathrm{~V}$. The integration time of the PD was $4.6 \mathrm{~ms}$.

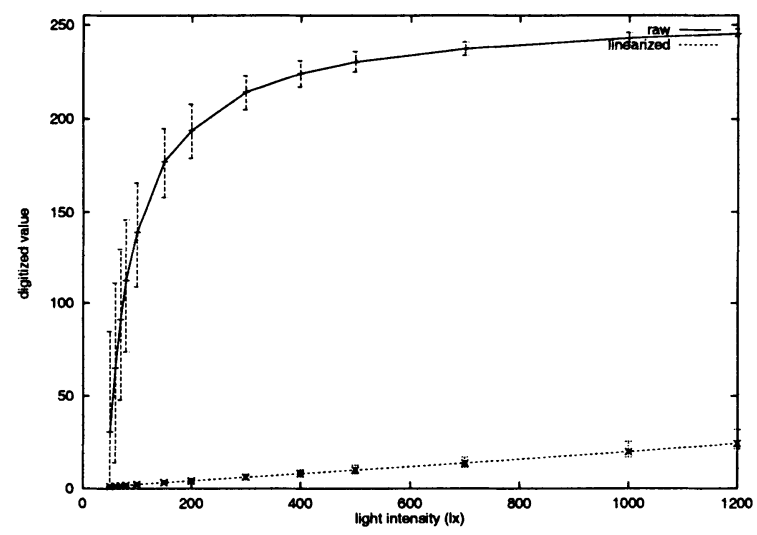

Figure 6. Sensor Characteristics

The graph shows that the average of output is a smooth function of light intensity. Owing to the $\mathrm{AD}$ conversion method of time counting, the output value is not linear with light intensity. But the output, which is linearized using the conversion equation $\mathrm{p}^{\prime}=\mathrm{pmax} /(\mathrm{pmax}-\mathrm{p})$, shows good linearity. From the difference between the maximum (or minimum) and average, effective resolution is about $3 \mathrm{bit}$. The main reason for the variation at the same intensity is fixed pattern noise (FPN) owing to the variation of transistor characteristic. As FPN appears as the offset of voltage, the effect is strong at low values when it is transferred to the time scale by $A D$ conversion. 
To evaluate the quality of image quantitatively, we calculated the rootmean-square (RMS) noise using the expression below. Figure 7 shows the graph of the RMS noise.

$$
R M S=\sqrt{\frac{\Sigma\left(p_{i}-\hat{p}\right)^{2}}{\Sigma p_{i}^{2}}} \times 100[\%]
$$

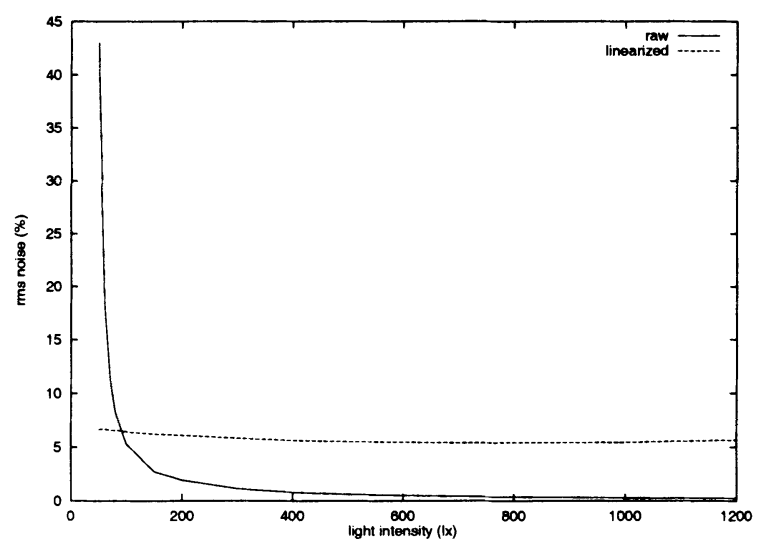

Figure 7. RMS Noise of the Sensor

This graph confirms that the FPN's effect is strong at low values. When the scale is linearized, the FPN's effect is almost flat at any value.

From the result of these experiments, the sensitivity and resolution are not particularly good, but still can be used in some applications.

It is proved that the $\mathrm{AD}$ conversion method used in the $\mathrm{PD}$ is weak against FPN as the voltage is transferred to time.

Overcoming the quality-of-image degradation of FPN, may be accomplished with the introduction of a good PD circuit with pixel-level AD conversion such as Yang et al. has proposed[11].

In this time, however, we tried to reduce the noise only by software. The program to download the offset and correct the input value by the offset is implemented. As mentioned above, the FPN's effect is strong at low values. It is proved theoretically and practically that the effect is proportional to the reversed input value. So the program calculates the product of the reversed input value and the offset and then subtract it from the input value. All of these calculations are processed in the vision chip. 
Figure 8 shows the result of the software noise reduction. The chip was flatly irradiated by an LED array lighting without a lens. The image was captured in 8 bit gray-scale and Vth was set to $2.4 \mathrm{~V}$. The integration time of the PD was $4.6 \mathrm{~ms}$. The offset was made from the output of 70lx irradiation. The images in Figure 8 is the output of 100lx irradiation.
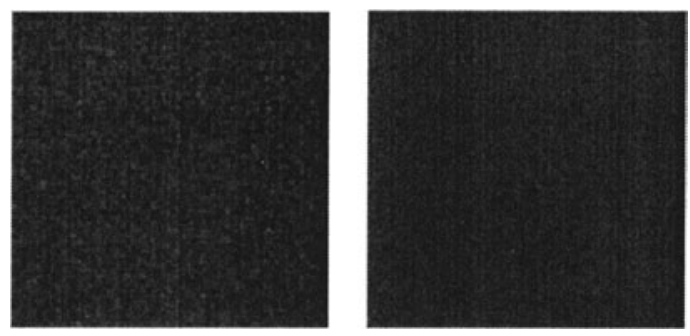

Figure 8. The Result of Software Noise Reduction (left: before correction, right: after correction)

Figure 8 shows the result of the software noise reduction. The chip was flatly irradiated by an LED array lighting without a lens. The image was captured in 8 bit gray-scale and Vth was set to $2.4 \mathrm{~V}$. The integration time of the PD was $4.6 \mathrm{~ms}$. The offset was made from the output of $701 \mathrm{x}$ irradiation. The images in Figure 8 is the output of 100lx irradiation.

\section{FURTHER INTEGRATION}

Semiconductor technology has been progressed rapidly in recent years and it is expected that it will continue to progress in the future. It is estimated in the technology roadmap that the gate length of the transistor will decrease by one-half in five years and the number of transistors that can be integrated in a unit area will increase. Also chip size tends to increase and the number of transistors that can be integrated in one chip will go to the order of Giga. In the future it is expected that Mega-pixel vision chips can be easily realized.

However, as the semiconductor process goes to a smaller scale, some design problems will appear. The problems in the case of further integration are examined in this section.

Concerning the effect on logic circuits, the existing design as it is can be shrunk with a process scale. For example, the circuit designed on the basis of $0.8 \mu \mathrm{m}$ process can be shrunk in $0.35 \mu \mathrm{m}$ process to $(0.35 / 0.8)^{2}$, or about $1 / 5$ th the area. Moreover, with increases in routing layers, more integration can be realized by wire globalization as descrived in section 4 . According to the well-known scaling rule, if the device size is $1 / \mathrm{K}$ and the power supply 
voltage is $1 / \mathrm{K}$, the power consumption will be $1 / \mathrm{K}^{2}$ and the the number of devices that can be integrated in a unit area will be $\mathrm{K}^{2}$ so the power consumption per unit area will be constant. But, as it is difficult to decrease the power supply voltage following the scaling rule, the power consumption will increase. Additionally with the increase in chip area and clock frequency, the power consumption will increase exponentially. This increased power consumption may have a bad effect on a vision chip integrated with image sensor. Therefore the architecture that tends toward low power consumption will be desired.

Concerning the effect on sensor circuits, using a standard CMOS and submicron process, some problems such as an increase in leakage current and decline in sensitivity will appear[12]. Therefore, the process modification will be needed. Moreover, with deep submicron processes, the power supply voltage will decrease according to the scaling rule and parasitic current will increase exponentially. The problem of power supply voltage is especially serious for with mixed-signal circuits. With a smallscaled process, a photodiode can be shrunk similar to other circuits, but with the limit of optical diffraction, the minimum size is about $4 \mu \mathrm{m}$. Figure 9 shows the shrink trend of the vision chip circuits.

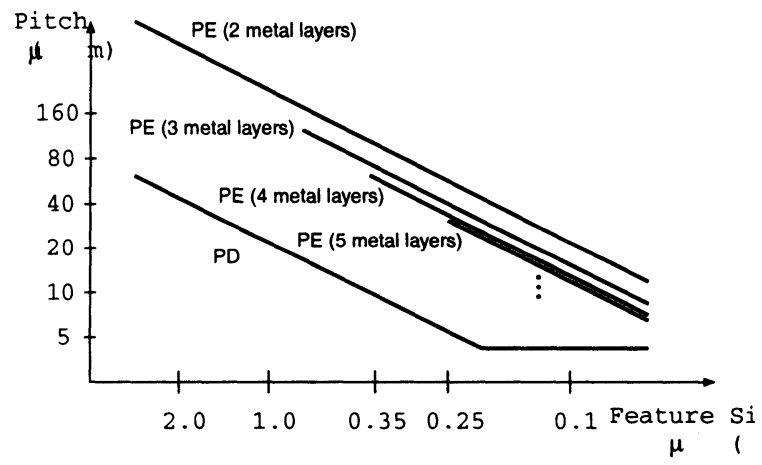

Figure 9. Circuits Shrink with Processing Scaling

\section{CONCLUSION}

We have designed the vision chip architecture for high speed image processing. Based on this architecture a $64 \times 64$ pixels prototype chip has successfully been developed. Further integration of vision chips in the future is also estimated. This vision chip will link image processing and real-time 
control, and open a new world of robot vision, intelligent transportation systems and many other applications

\section{REFERENCES}

[1] Alireza Moini : VISION CHIPS, Kluwer Academic Publishers (2000)

[2] M.Ishikawa, A.Morita, and N.Takayanagi : High Speed Vision System Using Massively Parallel Processing, Proc. Int. Conf. on Intelligent Robots and Systems, pp.373- 377 (1992)

[3] Y.Nakabo, and M.Ishikawa : Visual Impedance Using $1 \mathrm{~ms}$ Visual Feedback System, Proc. IEEE Int. Conf. Robotics and Automation, pp.2333-2338 (1998)

[4] Hiromasa Oku, Idaku Ishii, and Masatoshi Ishikawa: Tracking a Protozoon Using High-Speed Visual Feedback, Proc. of 1st Annual Int. IEEE-EMBS Special Topic Conf. on Microtechnologies in Medicine \& Biology, pp.156-159

[5] Akio Namiki, Yoshihiro Nakabo, Idaku Ishii, Masatoshi Ishikawa: 1ms Sensory-Motor Fusion System, IEEE Trans. on Mechatoronics, Vol.5, No.3, pp.244-252 (2000)

[6] M.Ishikawa : 1ms VLSI Vision Chip System and Its Application, Proc. IEEE Int. Conf. on Automatic Face and Gesture Recognition, pp.214-219 (1998)

[7] T.M.Bernard, B.Y.Zavidovique and F.J.Devos : A Programmable Artificial Retina, IEEE Journal of Solid State Circuits, Vol.28, No.7, pp.789-797 (1993)

[8] J.E.Eklund and C.Svensson and A.Astrom : VLSI Implementation of a Focal Plane Image Processor -- A Realization of the Near-Sensor Image Processing Concept, IEEE Trans. VLSI Systems, Vol.4, No.3, pp.322-335 (1996)

[9] Takashi Komuro, Idaku Ishii, and Masatoshi Ishikawa : Vision Chip Architecture Using General-Purpose Processing Elements for $1 \mathrm{~ms}$ Vision System, Proc. Int. Workshop on Computer Architecture for Machine Perception, pp.276-279 (1997)

[10] M.Ishikawa, K.Ogawa, T.Komuro, and I.Ishii : A CMOS Vision Chip with SIMD Processing Element Array for $1 \mathrm{~ms}$ Image Processing, Proc. IEEE Int. Solid-State Circuits Conf., pp.206-207 (1999)

[11] David X.D. Yang, Boyd Fowler and Abbas El Gamal, A Nyquist-Rate Pixel-Level ADC for CMOS Image Sensors, IEEE Journal of Solid-State Circuits, Vol.34, No.3, pp. 348-356 (1999)

[12] Hon-Sum Phillip Wong and Abbas El Gamal : Single-Chip CMOS Imaging Systems, 1999 ISSCC Tutorial (1999) 